\title{
Inter-organizational Negotiation of Web-services
}

\author{
Gabriel Costa Silva ${ }^{1}$, Itana Maria de Souza Gimenes ${ }^{2}$, Marcelo Fantinato ${ }^{3}$, Maria \\ Beatriz Felgar de Toledo ${ }^{4}$ \\ ${ }^{1}$ University of York, ${ }^{2}$ State University of Maringa, ${ }^{3}$ University of Sao Paulo, \\ ${ }^{4}$ University of Campinas \\ 1'gabriel_costasilva@yahoo.com.br, ${ }^{2}$ itana@din.uem.br, ${ }^{3}$ m.fantinato@usp.br, \\ ${ }^{4}$ beatriz@ic.unicamp.br
}

\begin{abstract}
The web service technology allows organizations to interact through business processes. However, organizations involved in cooperative business processes have different interests and points of view. A negotiation allows them to discuss their interests and requirements in order to reach an acceptable agreement. We propose an integrated web service negotiation process that takes into account human interaction and the use of different negotiation protocols. It focuses on the application of feature modelling to describe the negotiated services. Our contributions include: (i) the definition of a negotiation process; (ii) the definition of a conceptual model to support the negotiation of web services; (iii) reuse of artefacts generated throughout the negotiation process; (iv) coverage of critical elements in the negotiation of electronic contracts, such as role, properties of electronic services and contract models; and $(v)$ exploration of the process in different application scenarios.
\end{abstract}

Keywords: Business Process, Inter-organizational cooperation, Electronic Contract, Electronic Negotiation, Web Service, Quality of Service (QoS)

\section{Introduction}

Organizations around the world are looking for innovation and efficiency to ensure competiveness. Global cooperation has been considered a key factor in this context. Business process outsourcing, along with internet technology offer computing support that enables organizations to find partners, undertake negotiations and monitor the quality of contracted services even beyond geographical, cultural and technological limits [1, 2]. The web service technology, together with business process management concepts and tools offer a set of resources and standards that facilitate inter-organizational cooperation, such as those performed in supply chains $[3,4]$. The negotiation process for e-contract establishment which involves Web services, is one of the challenges that have been strongly discussed in literature [5]. Prior to the service invocation, both service provider and service consumers need to negotiate to achieve a mutual agreement [6]. However, according to Giambiagi et al., [5], the Web service model as well as its specification stack, do not widely support the concept of contracts. In addition, Grefen et al., [1] claims that Web services also does not widely support negotiation. Even though several languages have been used to enable negotiation and contract establishment, such as ebXML, WS-Agreement and WSLA [5], those languages do not support more sophisticated negotiations [7], such as that involving different negotiation protocols, or non-technical Quality of Service (QoS) attributes.

Even though several models [8], frameworks [9] and support systems [10] for enegotiation have been proposed, there is a lack of support to negotiation of e-services 
involving human interaction in the context of the web service technology. This paper presents a process that supports dynamic negotiation of e-contracts involving web services (WScontracts) as an approach to support inter-organizational cooperation. The process takes into account interaction amongst human negotiators and the use of different negotiation protocols. In order to realize the process, a prototype of a computer-aided support environment was developed and used to perform negotiations as well as to conduct a feasibility study.

The proposed negotiation process is provided within the context of a well-defined approach, called PL4BPM - Product Line for Business Process Management [11], and its respective support environment, called FeatureContract. This approach supports the representation of variability in business processes and web services through feature modelling, and the monitoring of WS-contracts throughout the process execution. PL4BPM is designed to model the artefacts involved in the negotiation amongst organizations willing to establish a common WS-contract to regulate their cooperation.

This paper is structured as follows. We present the research background, which is mainly concerned with business process, e-contract, negotiation and PL4BPM. Next, the proposed WS-negotiation-process and its activities are presented and illustrated with snapshots of the developed prototype. We then present an experimental study developed to evaluate the proposed process. Finally, we analyse related works and present the conclusions.

\section{Background}

This section introduces the concepts necessary to understand our work. First, we briefly introduce web services and electronic contracts (e-contract). We also survey the concept of negotiation. Next, we show the Product Line for Business Process Management (PL4BPM).

\subsection{Web Services, e-contract and Negotiation}

Web services technology has been identified as the most promising technology for the implementation of Service-Oriented Computing (SOC). According to this technology, a software can be decomposed into self-contained, loosely coupled and language independent units [3]. Business process can be used to compose web services; to integrate systems including legacy ones; to compose complex applications through services grouping and coordination; and to establish partnerships in distributed system development. A business process consists of a set of tasks undertaken in a specific sequence to achieve a business goal [4]. It also represents constraints on activities execution order as well as possible interactions between them. Initiatives integrating the concepts from SOC, along with the Business Process Management (BPM) have been shown benefits for a variety of companies [12].

An e-contract is a document used to represent an agreement amongst parties which is basically composed of: product or service definition; rights, obligations and prohibitions; and actions to be taken in case of disagreements. Contracts can be complex and their establishment process is often cumbersome due to the large number of parameters involved in the selection of QoS attributes and levels. Thus, a negotiation amongst the parties is necessary to define the issues involved in establishing an e-contract [1].

Negotiation is an interaction process amongst two or more partners in which their goal is to reach a mutually acceptable agreement $[13,14]$. Electronic negotiation (e-negotiation) is the process of conducting negotiations amongst business partners using electronic means [15]. Thus, a negotiation: (i) is defined as a process that contains the sequence of activities needed to start, conduct and finish a negotiation; (ii) involves, at least, two partners; (iii) requires interaction amongst partners; and, (iv) demands decision making. This process involves: 
negotiation protocols, negotiation objects and decision models [16]. Participants involved in a negotiation can be classified according to [13]: the level of automation, role and cardinality.

The level of automation varies from human support, to autonomous agents. The role defines the capabilities of a participant, such as a negotiation driver, the responsible to define the rules and conduct the negotiation, or a mediator, someone who intermediates the negotiation, acting up on behalf of one of involved parties. Information about a negotiation can be public or private according to the access level of the roles [14]. The interactions amongst partners are guided by the rules of the negotiation protocols [17]. These rules define how the partners exchange offerings and decide on how to proceed or end a negotiation. Decision making is an internal and private process based on a cost-benefit model or strategy. The decision making depends on the negotiation strategy used [6]. In the e-service context, the negotiation process is a requirement to reach an agreement and then establish an econtract $[1,18]$.

\subsection{Product Line for Business Process Management (PL4BPM)}

PL4BPM aims to provide support both to model variability in business processes and web services, and to monitor WS-contracts throughout the process execution [11]. It is designed to model the artefacts involved in the negotiation amongst organizations willing to establish a WS-contract to regulate their cooperation. The overall framework of PL4BPM is presented in Figure 1 using a UML diagram. The architecture of such approach is in compliance with the reference architecture for e-contracts proposed by [19].

The approach comprises four organizations: Consumer, Provider, Negotiator and Monitor. The Consumer Organization includes the following structures: (i) a structure called WSContract Definition responsible for the establishment of WS-Contracts based on feature models; (ii) a structure called WS-Contract Execution responsible for the business process execution; and, (iii) a SOC System necessary if the consumer services are part of the business process to be executed. In the Provider Organization(s), the SOC System controls the web services subcontracted by the consumer. The Monitor Organization has one structure, the WS-Contract Monitor that follows the business process execution guided by the QoS terms contained in the WS-contract for service monitoring. The Negotiator Organization has the WS-Contract Negotiation package that uses a set of protocols responsible for negotiation of WS-contracts between consumers and providers.

Feature modelling allows the representation of e-services and possible levels for QoS attributes. A feature model is represented through a tree-like diagram [20]. In this paper, they are graphically represented in the style of the FeaturePlugin tool. In addition, the use of Product Line (PL) concepts brings additional benefits to inter-organizational business process, such as dynamism and flexibility [21]. We have developed a feature metamodel in which the e-services feature diagram consists of two sub-trees, identified by the following pre-named root features: (i) e-services sub-tree that contains the features representing the eservices provided by an organization; and, (ii) qos-attribute sub-tree that contains the features representing the QoS attributes and levels which can be associated to the e-services. Examples of feature models used to represent e-services are presented throughout the negotiation process description in the following section. 


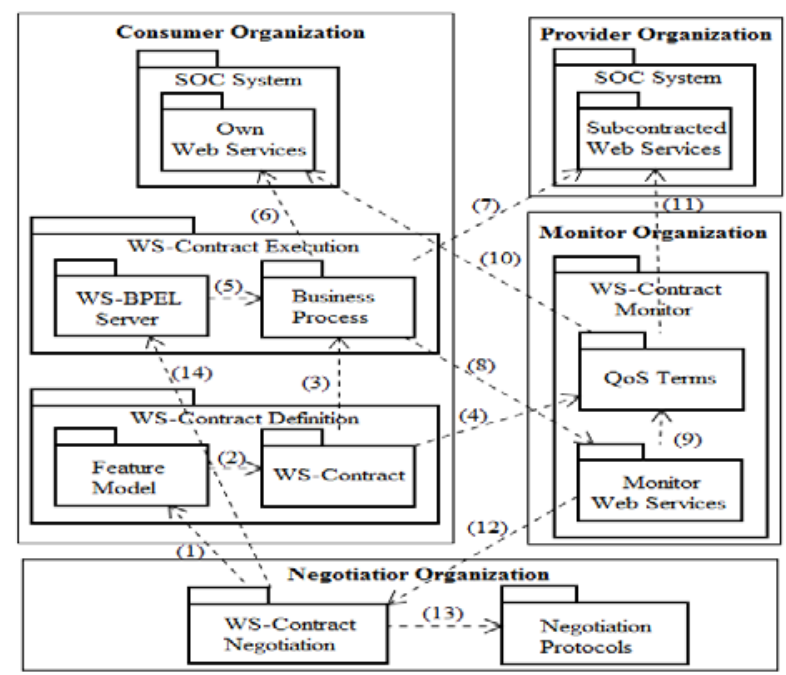

Figure 1. PL4BPM Execution Environment Architecture

\section{WS-negotiation Process}

The WS-negotiation process proposed in this paper has the following principles: (i) it focuses on dynamic negotiation amongst organizations which are interested in undertaking a collaborative business process composed of web services; (ii) it promotes the reuse of the core artefacts produced throughout the negotiation processes; (iii) it supports decision making throughout the negotiation; (iv) it supports different negotiation protocols; and, (v) it takes into account interaction amongst human negotiators. In the context of this work, artefacts refer to the feature model, electronic contract, and the entire negotiation base, including, for instance, negotiation roles and negotiation threads. These artefacts are created throughout the first lifecycle of negotiation process.

The WS-negotiation process is carried out according to the PL4BPM environment architecture, as described in Figure 1. The numbers in the figure represent indexes to explain the dependences amongst packages. In this paper, we analyse only the relations relevant to the WS-negotiation. Fantinato, Gimenes and Toledo [11] provide overall information about the PL4BPM approach. The WS-Contract Negotiation package comprises the WS-negotiation process and its related elements. To perform a negotiation, the package depends on the Negotiation Protocols package (13), which describes the rules for different types of negotiation (e.g., bargain, auction, and so on). The negotiation also depends on the Feature Model(s) (1) in the WS-Contract Definition, which is responsible to provide the elements to define the e-services, its properties, and QoS attributes. The electronic contract yielded as the result of a negotiation, is deployed on the WS-BPEL Server (14). Finally, the negotiated QoS attributes are monitored by Monitor Web Service (12).

The WS-negotiation-process was mainly based on the negotiation framework proposed by Kim and Segev [22] and the process model for e-negotiation proposed by Kersten et al., [23]. The process consists of eleven activities, as shown in Figure 2, divided into two life cycles: the Planning and Negotiation Agenda Settings and the Negotiating and Establishing WSContract. Whereas Figure 1 shows the packages of the PL4BPM architecture, and the Figure 3 shows the conceptual entities in these packages, the Figure 2 focus on the negotiation process. Each activity in the process uses different entities to fulfill their duties. The first cycle defines the elements of the negotiation base, which are: (i) the negotiator roles, set up in activities 2 and 5; (ii) the business partners involved, set up in activities 5 and 6; (iii) target 
services of the negotiation, set up in activities 3, 4 and 6; (iv) the negotiation variables for each service and their respective possible values, set up in activities 3 and 6; and, (v) the econtract template, set up in activity 7. In the second cycle, the actual negotiation amongst the parties takes place by selecting the offered services (activity 8), negotiating the variables of these services (activities 9 and 10) and finally, establishing the WS-contract (activity 11).

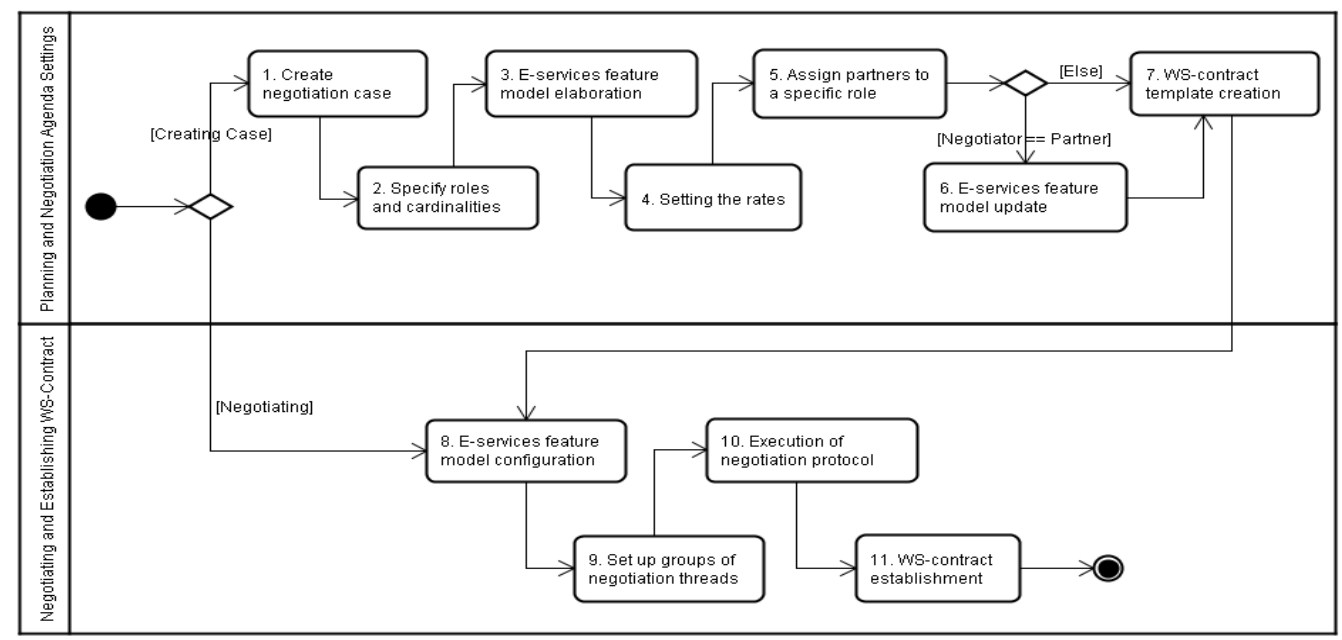

Figure 2. WS-Negotiation Process Model

\subsection{Example on Acquisition of Services in the Distributed Development of Software}

The example scenario used to present the WS-negotiation-process is the Distributed Development of Software (DDS) for a hotel chain. There are three types of organizations involved: (i) the hotel chain that contracts Organization A to develop the software; (ii) Organization A, responsible for the software development which might involve subcontracting partners to support and enhance the development; (iii) partner organizations, responsible for developing specific parts of the software according to an established contract. This example concerns the negotiation process carried out between Organization A and the providers B, C ... N. Organization A is contracted to develop a reservation system. In order to improve its development strategy, it decides to subcontract, for example, the development of the GUI (Graphical User Interface) during the implementation phase. All the communication amongst Organization A and its partners will be undertaken through web services. From the SOA perspective, Organization A is a consumer of services whereas the Organizations B, C $\ldots \mathrm{N}$ are providers. From the negotiation perspective, Organization $\mathrm{A}$ is as a negotiation driver; thus, responsible for structuring and conducting the negotiations whereas the other organizations are viewed as negotiation partners.

\subsection{Negotiation Conceptual Model}

The negotiation involves several related entities that compose the negotiation base. They are persistent elements defined in the first negotiation life cycle and used in the second life cycle to undertake the actual negotiation. Moreover, the negotiation base can be reused in further negotiations. The entities of the conceptual model are presented in Figure 3. These entities are distributed amongst the packages presented in Figure 1. This model is an important contribution of our work. 


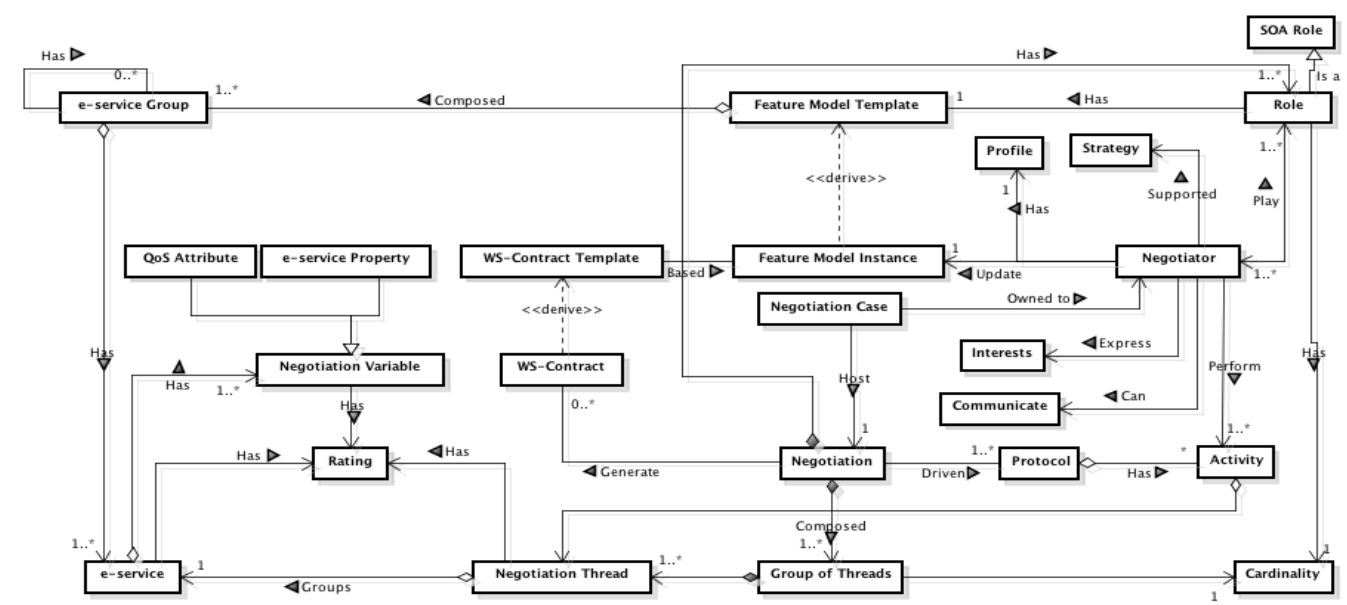

Figure 3. The Conceptual Negotiation Model

A Negotiation is conducted within a Negotiation Case, which is the main element of the WS-negotiation-process. A Negotiation Case belongs to only one Negotiator who is its owner. A Negotiator is a human, in this case, a person commissioned to act up on behalf of the company he/she represents. Each Negotiator has several Roles. An invited Negotiator assumes a Role within a Negotiation, which defines its responsibility, such as "Credit Verification Service Provider". It is a specialization of the SOA roles, thus an organization can be a consumer or a provider, for example. The Role cardinality defines the number of negotiators, in that role, that can win the negotiation to provide and consume services at the end of the negotiation. For example, in a negotiation for a tourism package, two airlines can win a trip, one to go and another to return, thus the cardinality is 2 (two). A Feature Model Template, associated with a Role, defines groups of e-services (e-service Group), which are related to Negotiation Variables. These variables represent QoS Attribute(s) and e-service Property(ies).

A Negotiator is invited to participate of a Negotiation, playing a certain Role, based on a Profile, containing personal information. Each Negotiator receives a Feature Model Instance, updated according to the services that it can offer. According to the Negotiator interests, a Rating can be assigned to each e-service and Negotiation Variable. This Rating is part of the information base used by negotiators to define the negotiation Strategy. The Negotiator (consumer) expresses its interests by configuring the Feature Model Instance of its partner (provider).

The Negotiation is guided by a Protocol that defines a set of Activity(ies), like making offers and counter-offers. Negotiation Thread(s) are formed of e-service Group(s) provided by a negotiation partner. A Negotiation Thread has a Rating. A Group of Thread has a Cardinality. A successful Negotiation produces one or more WS-contracts based on a WScontract template.

\subsection{Activities of the Planning and Negotiation Agenda Settings Life Cycle}

Carried out by the negotiation driver (an instance of Negotiator), in this life cycle the negotiation base is created. The artefacts related to the negotiation base (e.g., Negotiation threads), are reused in the second life cycle.

(1) Create Negotiation Case: this activity defines the objective of the negotiation and creates the negotiation case. The elements of a WS-contract negotiation are grouped within a 
negotiation case [24]. The attributes of a negotiation case includes: a title, such as "Webbased graphical user interface", a brief description of the negotiation case that can be used in a directory where partners can find negotiations of their interest; keywords; privacy information of the case; and the deadline to end the negotiation in order to proceed to the WScontract establishment.

(2) Specify Roles and Cardinalities: this activity creates the roles played by negotiators throughout a negotiation. The organization partners act within specific roles. A role groups a set of partners capable of providing the same type of service. In the same negotiation case there might be several roles. Our scenario example has two main roles: i) the Reservation software development organization playing the role of consumer; and ii) the Web-based UI development Organization playing the role of provider. This activity defines the negotiation direction, i.e., if the consumer searches for providers or the providers search for consumers. In our example, the negotiation direction represents a consumer looking for providers. Finally, cardinality has to be defined to each role. In our example, the role assigned to the consumer organization has cardinality 1 (one) whereas the provider has cardinality 999 (infinite). This allows the consumer organization to acquire services provided by different organizations.

(3) E-services Feature Model Elaboration: e-services to be contracted are represented in the feature model as a template, which is created in this activity. The created model represents a set of services that the respective negotiator can offer. The feature model is the key enabler for the artefact reuse. Once the feature model represents the services offered, it can be reused several times to conduct negotiations in the second life cycle.

The feature model is assigned to a role instead of a specific negotiator once the possible negotiators are not still known in this activity. The model can have one or more associated negotiation variables. A negotiation variable represents an issue associated to the service that needs to be negotiated. It is an item of evaluation that supports the negotiator. Price is an usual example of negotiation variable [25]. PL4BPM considers two main types of negotiation variables: e-services properties and QoS attributes. In our example scenario, e-services properties represent additional characteristics and/or specific requirements, such as an option in the control menu. QoS attributes represents non-functional requirements such as access control. Figure 4 represents a sub-tree of the feature model (FM) of the Web-based UI development Organization.

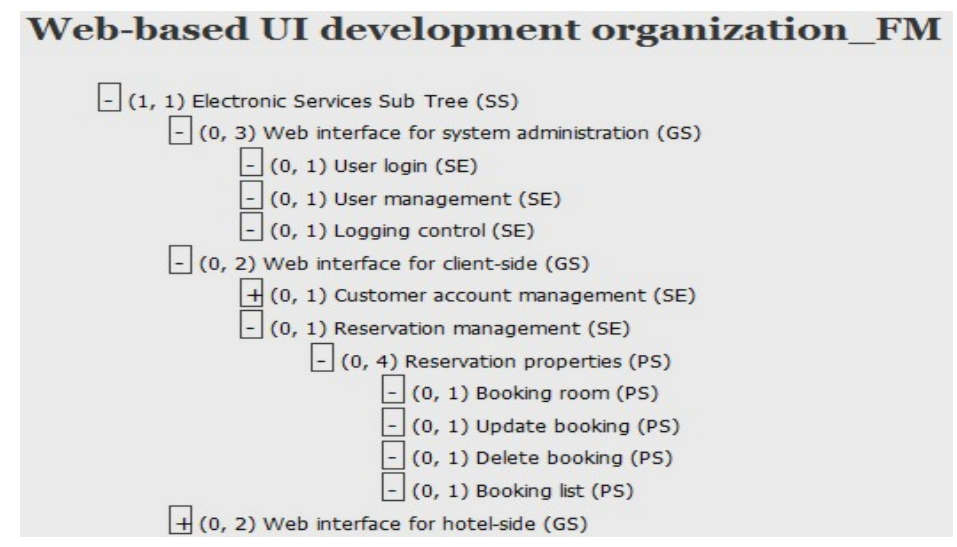

Figure 4. Excerpt of a Feature Model - GS(Group of Services); SE (Service); PS (Property of a Service) 
The Electronic services Sub Tree contains the services that can be offered by the providers. There are three groups of services: Web interface for system administration; Web interface for client-side; and Web interface for hotel-side. In the Reservation management we can see defined properties such as: Booking room, Update booking, Delete booking and Booking list. Each element of the feature model has an associated pair of values that defines it as optional or mandatory. For example $(0,3)$ means optional and maximum value 3 . This is because the group has three services that can be selected. As another example, $(1,1)$ means mandatory and maximum value 1 . This representation is in line with feature modelling literature [20].

The QoS Attributes Sub Tree contains attributes such as security and access control, as shown in Figure 5. Each attribute can be associated with levels of controls. In our scenario, the QoS attributes represent non-functional requirements of the graphical interface, like Access control with User auth (authentication). The QoS attributes are then associated with respective services to which they apply using references.

(4) Setting the Rates: negotiators have different interests, whereas offering a service may be an advantage for a negotiator, it might not be for another. Thus, rates represent the importance of each negotiation item for a negotiator. They are assigned to: group of services, services and negotiation variables. The rates of a group of services vary from 0 (lowest) to 100 (highest). The rate of a service varies from 0 to the rate value of its group. After rating, we can see the importance of each service for the involved negotiators. Table 1 presents a hypothetical rating for organizations B, C and D according to the services and negotiation variables provided.

\section{Web-based UI development organization_FM}

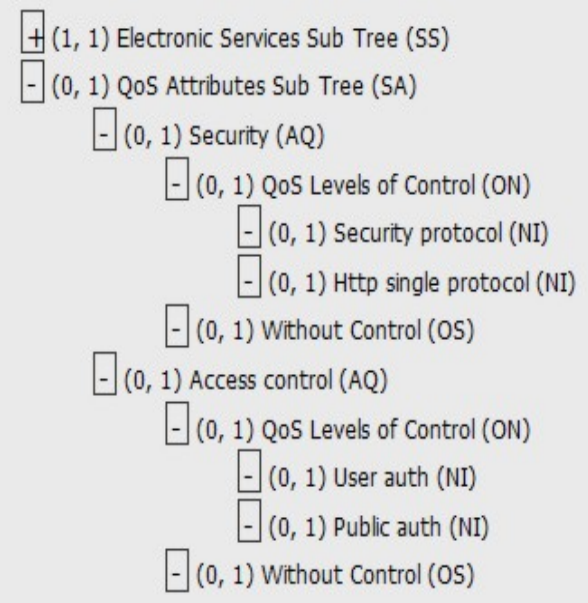

\section{Figure 5. Excerpt of a QoS Sub Tree - AQ (Attribute of QoS); OS (Option - NoLevels); ON (Option - Levels); NI (Level of QoS)}

At this moment, only the negotiation driver (i.e., the one that creates the negotiation case) is capable of rating the items of its interest because the negotiators were not invited yet. When further negotiators start their participation, each of them has to assign rates to its feature model. These rates are strategic information because they directly express the negotiator preferences. Thus, they are private information that cannot be shared amongst negotiators. They are used to support the selection of the offers. In addition, once the rates are assigned to group of services, services and negotiation variables, it is also reused in future negotiations. 
Table 1. Rates Assigned by the Negotiators

\begin{tabular}{lccc}
\hline & $\begin{array}{l}\text { Web interface } \\
\text { for system } \\
\text { administration }\end{array}$ & $\begin{array}{l}\text { Web } \\
\text { interface for } \\
\text { client-side }\end{array}$ & $\begin{array}{l}\text { Web interface } \\
\text { for hotel-side }\end{array}$ \\
\hline Organization B & 100 & 80 & 30 \\
Organization C & Not provided & 80 & 80 \\
Organization D & 45 & 100 & 20 \\
\hline
\end{tabular}

(5) Assign Partners to a Specific Role: SOA partners are consumers and providers. In our example, the organization that develops the reservation software is the consumer, and organization B, C and D, are providers. Negotiators are invited when they are previously known or they can be found in public directories. They can also offer themselves to participate of a negotiation when a call is made. When a negotiator is associated to a role, it receives the feature model instance based on feature model template for that role.

(6) E-services Feature Model Update: a partner may either not be capable of providing the services or even provide additional services. Thus, it is necessary that the provider organization review its feature model instance to represent its capabilities. In the negotiation literature, this revision is usually part of the negotiation agenda configuration [23]. It may demand modifications in the group of services, services and negotiation variables according to provider organizations. In our example scenario, consider for instance, the group of services named Web interface for system administration. Suppose that, for some reason, one of the providers cannot offer the interface according to the defined requirements. This organization then withdraws this group of services from its feature model instance. Figure 6 shows this updating in contrast with the feature model presented in Figure 4.

(7) WS-contract Template Creation: this activity creates the WS-contract template that contains information that can be used in any similar contract established from the defined feature model.

\subsection{Activities of the Negotiating and Establishing WS-contract Life Cycle}

The negotiating and establishing WS-contract life cycle uses the structure defined in the first life cycle to support the negotiation amongst partners. In this life cycle, the services offered are selected to be contracted, the negotiation protocol is carried out and the WScontract is established. This life cycle might be carried out several times. Each execution of this life cycle represents a negotiation amongst the involved parties. Thus, the artefacts previously defined are reused although some updates might be required (e.g., new organizations participating in the negotiation). 
(10) Execution of Negotiation Protocol: a negotiation protocol defines both style and rules that guide the bidding process. The negotiation proceeds or ends according to these rules. Our negotiation process can support the most common negotiation styles and allows the protocol to be chosen dynamically. During the negotiation protocol execution, each service needs to be negotiated aiming at reaching an agreement amongst partners. By following the rules and observing the cardinalities, the winners of each negotiation thread are selected. The players can exchange shorts messages throughout the negotiation process. In our example, we have a dispute amongst organization $\mathrm{C}$ and $\mathrm{D}$ to provide the Reservation management service, considering that the cardinality to this group of negotiation thread is 1 (Figure 8).

In such a case, the consumer can choose to use the bargain protocol to negotiate with these organizations. The consumer can make a new proposal to both organizations and wait for a response. On the other hand, these organizations can make a counter-offer. The negotiation game will continue until the consumer and one provider reach an agreement. The amount of providers that can provide a group of services is defined by the cardinality of the role and the group of negotiation thread.

(11) WS-contract Establishment: having defined the services and providers, the last activity can be executed. It consists of generating an instance of the WS-contract based on the template defined in first cycle of the negotiation process. The final WS-contract contains the service contracted by each winner organization of the negotiation.

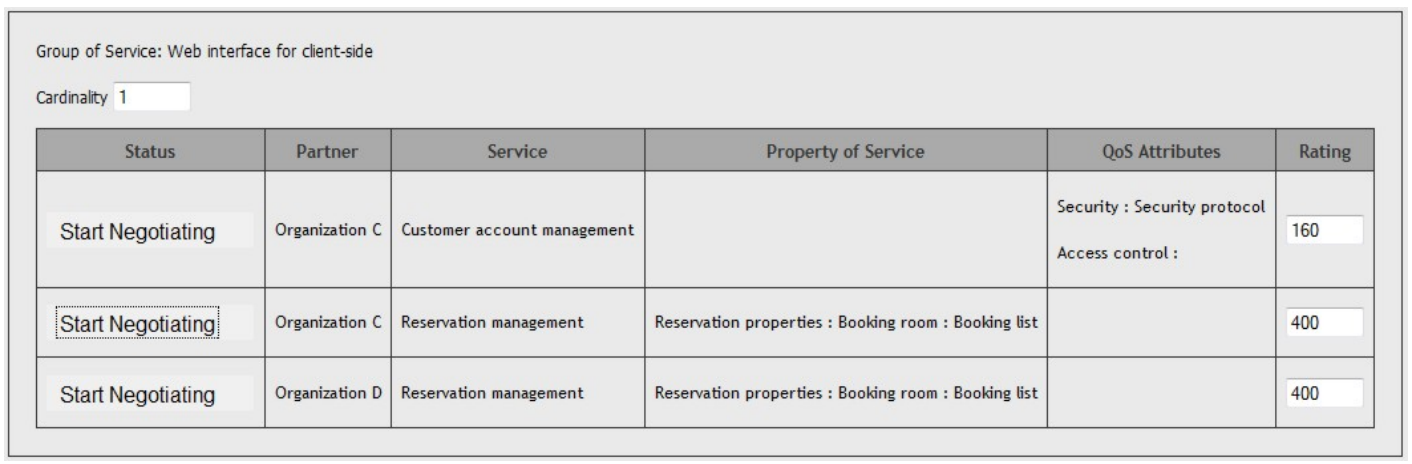

Figure 8. Group of Negotiation Thread for Web Interface for Client-side

\section{Experimental Study}

An experimental study was carried out to analyse the WS-negotiation process with the purpose of providing evidence of its feasibility and usage relevance. The study was conducted accordingly the guidelines proposed by Gonçalves et al., [26] for experimental studies in PL4BPM context. A computer-supported prototype was developed to automatize the proposed negotiation process. The prototype provides a portal through the partners can find each other, set their interests and conduct a negotiation. The prototype was used in the study to support the participants.

\subsection{Scenario Domain}

In this subsection, we explore a second scenario in which the WS-negotiation process was applied. Although it is a hypothetical case, the participants of this study acted up according to the roles defined in order to analyse the process feasibility. The case takes into consideration an inter-organizational business process involving four organization types: a travel agency, hotels, airlines and car rental companies. This scenario involves: (i) organizations, in which 
one is a consumer organization and three are providers; (ii) negotiators, people that represent organizations throughout the negotiation; and (iii) services offered, such as car rental.

From the WS-negotiation-process point of view, we consider that the travel agency is looking for providers to operate its business process, thus it will negotiate services to establish a WS-contract with selected providers. The specification of the services looked for the travel agency is provided by feature models. An example of the feature model provided for the specification of the Airline services is shown in Figure 9. This feature model specifies the group of service Ticket management with services: Seeking ticket, Buying ticket; and Cancel ticket.

These services have a QoS Attributes sub-tree (Figure 10). It has two QoS Security and Availability. The possible levels for the Security are authentication by user (User Auth) or public authentication (Public auth). The QoS attribute Availability has the following control levels: 24x7, 24x5 and $8 \times 5$ (hours $x$ weekdays). In our hypothetical negotiation scenario the travel agency acts in the role of consumer interested in contracting services from the providers. Several organizations act in the role of providers with the following cardinalities:

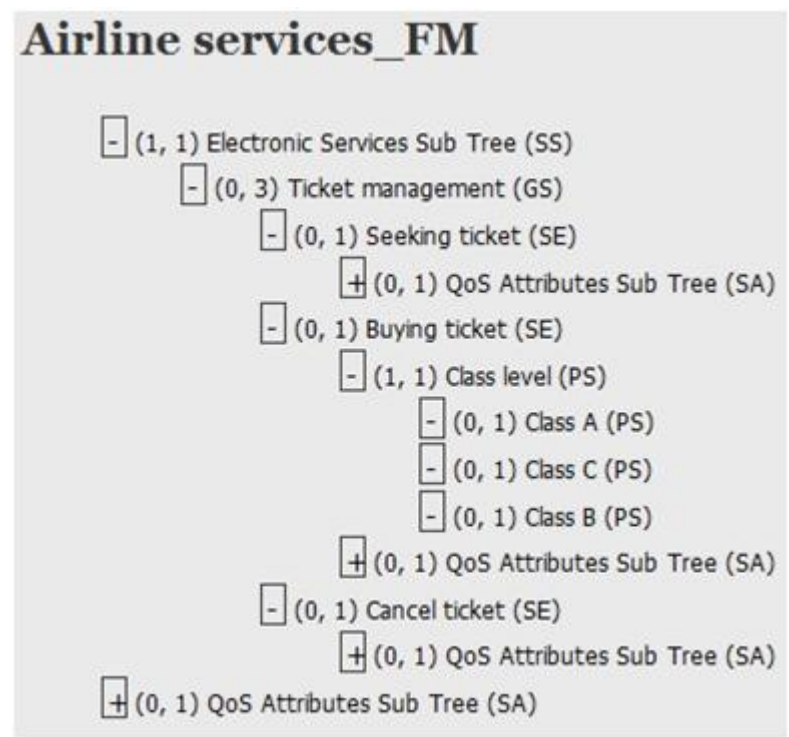

Figure 9. Feature Model for the Role of Airline Company

- Hotel: cardinality 1 (one) - the travel agency covers only one destination so it is interested in contracting one hotel;

- Airline: cardinality 2 (two) - the agency can hire one company to go and another to return;

- Car rental: cardinality 999 (any number) - the client can rent different cars for each occasion.

\subsection{Execution of the Experimental Study}

The experimental study involved 5 (five) participants who are post-graduation students in computer science. The participants had three hours of training, two for the negotiation process techniques and one for the domain. They were divided into 4 (four) groups, one group with two participants and 3 (three) with one. Each participant was representing a role for one 
organization; except for the car rental organizations to which we had two participants. The travel agency was the negotiation driver. The negotiation protocols were bargain, auction and fixed-price. The participants were allocated in one room, each in a computer running the prototype of the WS-negotiation process support environment. The participants executed the eleven activities of the process. At the end they filled a questionnaire.

\subsection{Discussion}

Data collected from the questionnaire were analysed regarding the feasibility and usage relevance of the WS-negotiation-process. Table 2 shows the analysed issues and the percentage of participant answers. Overall results show that participants were in favour of the WS-negotiation process feasibility and usage relevance. When asked if the activities were clearly defined, $100 \%$ of the participants have agreed. Nevertheless, only $20 \%$ of the participants found the WS-negotiation-process easy to apply. They all considered the training satisfactory but $40 \%$ of them required assistance during the negotiation process. We did not consider this relevant as the set of technological issues is really hard for beginners.

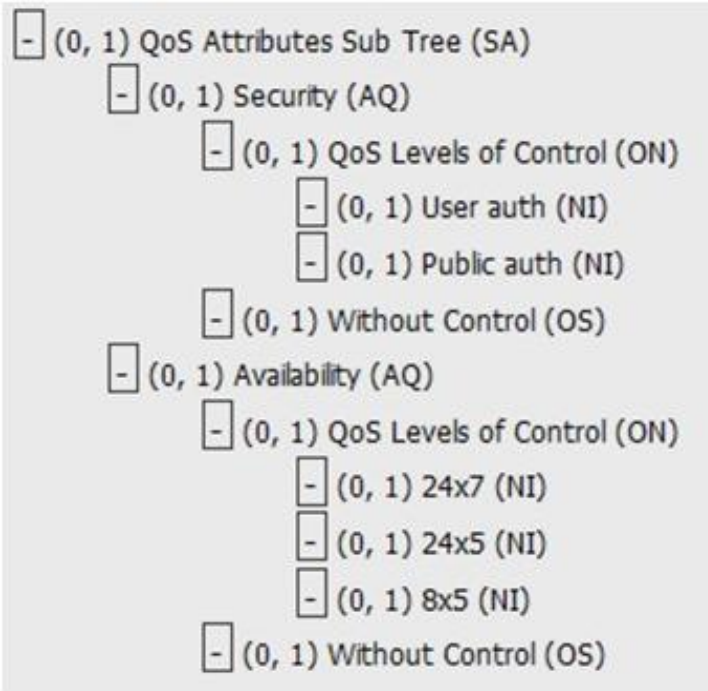

Figure 10. Feature Model Representing the QoS Attributes of Services

Participants were asked if they consider the WS-negotiation process feasible. $100 \%$ of them considered the process useful and relevant so they would apply it again. They confirm that there are advantages of using the process as compared to informal negotiation. $100 \%$ of the participants agreed that all the partners involved can have benefits from the process support. We considered the prototype a threat of validity to the experiment, as it provides only the basic functions and a simple user interface. Some difficulties faced by the participants were related to the prototype stage of the support environment.

A questionnaire was also applied to detect the experience of participants with the subject. Most of them have studied the subjects in the academic context but did not have previous contact with the involved concepts.

\section{Related Work}

Negotiation is a multidisciplinary area, thus it is discussed in Bichler et al., [27]: anthropology, management, psychology, economy and computer science. We could not find 
works that apply the same ideas of our approach towards modelling e-service negotiation. In particular, in the use of feature modelling to describe the negotiated services and its focus on artefact reuse. However, some works provided a solid background, we mainly point out: (i) the frameworks of Kim and Segev [17], Comuzzi et al., [28] and Mukhtar et al., [29]; (ii) the processes of Chiu et al., [30], Kersten et al., [23] and Elfatraty and Layzell [31]; and, (iii) the model of Lin [8] to support negotiation in electronic environment.

Table 2. Data Collected from the Participants of the Experimental Study

\begin{tabular}{|l|c|c|}
\hline \multicolumn{1}{|c|}{ Question } & Answer & (\%) \\
\hline \multirow{2}{*}{$\begin{array}{l}\text { Was the WS-negotiation process } \\
\text { training satisfactory? }\end{array}$} & $\begin{array}{c}\text { I agree, but I needed assistance during } \\
\text { the negotiation process }\end{array}$ & $40 \%$ \\
\cline { 2 - 3 } & $\begin{array}{c}\text { I agree. I did not need any assistance } \\
\text { during the negotiation process }\end{array}$ & $60 \%$ \\
\hline $\begin{array}{l}\text { Are the WS-negotiation activities } \\
\text { clearly defined? }\end{array}$ & I agree & $100 \%$ \\
\hline \multirow{2}{*}{$\begin{array}{l}\text { Is the WS-negotiation process easy } \\
\text { to apply? }\end{array}$} & I agree & $20 \%$ \\
\cline { 2 - 3 } $\begin{array}{l}\text { Is the WS-negotiation process } \\
\text { feasible to all the parts involved? }\end{array}$ & I agree & $80 \%$ \\
\hline $\begin{array}{l}\text { Is the WS-negotiation process } \\
\text { feasible and useful? }\end{array}$ & I agree. I would apply it again & $100 \%$ \\
\hline $\begin{array}{l}\text { Are there advantages of using the } \\
\text { WS-negotiation process when } \\
\text { compared to informal negotiation? }\end{array}$ & I agree, but it is hard to apply & $20 \%$ \\
\cline { 2 - 3 } & I agree & $80 \%$ \\
\hline
\end{tabular}

Table 3 highlights items that we use to compare related works to ours. The items are: application area, multi-parties, multi-protocols, multi-items, multi-variables, support to decision making, human interaction throughout negotiation, e-contracts, web services and reuse of artefacts.

Table 3. Features of Negotiation Processes

\begin{tabular}{c|c|c|c|c|c|c|c|c}
\hline & $(1)$ & $(2)$ & $(3)$ & $(4)$ & $(5)$ & $(6)$ & $(7)$ & $\begin{array}{c}\text { This } \\
\text { process }\end{array}$ \\
\hline Multi-parties & & $\mathrm{X}$ & $\mathrm{X}$ & $\mathrm{X}$ & $\mathrm{X}$ & $\mathrm{X}$ & & $\mathrm{X}$ \\
\hline Multi-protocols & & $\mathrm{X}$ & $\mathrm{X}$ & $\mathrm{X}$ & $\mathrm{X}$ & $\mathrm{X}$ & $\mathrm{X}$ & $\mathrm{X}$ \\
\hline Multi-items & & & & & & & & $\mathrm{X}$ \\
\hline Multi-variables & & $\mathrm{X}$ & $\mathrm{X}$ & $\mathrm{X}$ & $\mathrm{X}$ & $\mathrm{X}$ & & $\mathrm{X}$ \\
\hline $\begin{array}{c}\text { Support to decision } \\
\text { making }\end{array}$ & & $\mathrm{X}$ & & & & $\mathrm{X}$ & $\mathrm{X}$ & $\mathrm{X}$ \\
\hline $\begin{array}{c}\text { Human interaction } \\
\text { throughout negotiation }\end{array}$ & $\mathrm{X}$ & $\mathrm{X}$ & $\mathrm{X}$ & $\mathrm{X}$ & $\mathrm{X}$ & $\mathrm{X}$ & & $\mathrm{X}$ \\
\hline Contracts & $\mathrm{X}$ & & & & $\mathrm{X}$ & & $\mathrm{X}$ & $\mathrm{X}$ \\
\hline Web services & $\mathrm{X}$ & & $\mathrm{X}$ & & & & $\mathrm{X}$ & $\mathrm{X}$ \\
\hline Reuse of artifacts & & & & & & $\mathrm{X}$ & $\mathrm{X}$ & $\mathrm{X}$ \\
\hline
\end{tabular}


Lin [8] (1) presents a conceptual model to specify a negotiation process in a serviceoriented environment. The model defines a set of functionalities to each SOA element throughout the negotiation process as well as the interaction protocol amongst them. However, this model is limited to one provider and one consumer for each negotiation which constrains the specification of more complex negotiation such as supply chains.

Elfatatry and Layzell [31] (7) presents a negotiation process composed of three phases which defines favourite providers, roles and the establishment of a reliable negotiators base at the end of the process. However, details about the activities and artefacts are not provided.

Comuzzi et al., [28] (3) propose a framework that aims at discovering the negotiation protocol supported by the negotiators. It focuses on an important issue, the cooperation amongst partners, but it only deals with part of the negotiation process. Mukhtar et al., [29] (4) propose an integrated framework for electronic markets. Although it integrates technologies like internet and SMS to automate negotiation activities, it only deals with one scenario.

The Kim and Segev [17] (2) framework and the Kersten et al., [23] (6) process provide basis for the conception of e-negotiation systems. They provide support for negotiation in dynamic environments; however, they do not take into account WS-contracts neither specific issues of web services, such as QoS. Chiu et al., [30] (5) present a process and a metamodel for contract negotiation in B2B domain. However, it does not allow simultaneous negotiation of several items. Our negotiation process tackles the gaps of these works by taking into account web services negotiations which deals with: multi-parties, multi-protocols, multiitems, multi-variables, decision making, human interaction and artefact reuse.

\section{Conclusions}

This paper presents a negotiation process to support the establishment of WS-contracts involving business processes composed of web services as an approach to support interorganizational cooperation. The negotiation process takes place in an environment that facilitates artefact reuse based on product line and feature modelling concepts. The negotiation process is composed of two main life cycles: (i) the Planning and Negotiation Agenda Settings; and (ii) the Negotiating and Establishing WS-Contract. Whereas in the first life cycle the feature model and the entire negotiation base is defined, in the second life cycle these elements are used to support the exchange of bids, i.e., the negotiation. The artefacts, such as negotiation threads, can be reused to support countless other negotiations.

In addition, we presented a computer-supported prototype developed to support an experimental study, which aimed to provide evidences of the feasibility and usage relevance of the process. The results confirmed that the proposed process is feasible and its usage is relevant for all the roles involved. However, we understand that an experiment with specialists is needed. The contributions of this work include: (i) the definition of a negotiation process; (ii) the definition of a conceptual model to support the negotiation of e-services; (iii) reuse of artefacts generated throughout the negotiation process; (iv) coverage of critical elements in the negotiation of electronic contracts, such as role, features of electronic services and contract models; and (v) exploration of models in different application scenarios.

Future work includes the study of renegotiation in the context of the proposed conceptual model of negotiation. Although renegotiation is similar to negotiation in terms of roles, protocols, strategies and others, it appears more rarely in the literature. Vecchiato et al., [32] extended the WS-contract feature meta-model to contain actions and restrictions to contemplate renegotiation. Therefore, this new WS-Contract model will be further incorporated to our proposed negotiation conceptual model to deal with renegotiation. 


\section{References}

[1] P. Grefen, H. Ludwig, A. Dan and S. Angelov, “An analysis of web services support for dynamic business process outsourcing”, Information and Software Technology, vol. 48, no. 11, (2006), pp. 1115-1134.

[2] A. Sharma and P. Loh, "Emerging trends in sourcing of business services", Business Process Management Journal, vol. 15, no. 2, (2009), pp. 149-165.

[3] M. Papazoglou, "Web Services: Principles and Technology”, 1st ed. Harlow: Pearson-Prentice Hall, (2007), pp. 784.

[4] M. Weske, “Business Process Management”, 1st ed. Berlin: Springer, (2007), pp. 384.

[5] P. Giambiagi, A. P. Ravn, O. Owe and G. Schneider, "Language-Based Support for Service Oriented Architectures: Future Directions”, Proceedings of 1st International Conference on Software and Data Technologies (ICSOFT 2006), (2006), pp. 339-344.

[6] P. C. K. Hung, "WS-Negotiation: an overview of research issues", Proceedings of the 37th Annual Hawaii International Conference on System Sciences, (2004), pp. 1-10.

[7] M. Parkin, D. Kuo and J. Brooke, “A Framework \& Negotiation Protocol for Service Contracts”, 2006 IEEE International Conference on Services Computing (SCC’06), (2006), pp. 253-256.

[8] J. Lin, “A conceptual model for negotiating in service-oriented environments", Information Processing Letters, vol. 108, no. 4, (2008), pp. 192-203.

[9] O. Abass and G. Ghinea, "Electronic Negotiation Frameworks: A Snapshot of the State of the Art”, 2006 Innovations in Information Technology, (2006), pp. 1-6.

[10] G. E. Kersten and H. Lai, “Negotiation Support and E-negotiation Systems: An Overview”, Group Decision and Negotiation, vol. 16, no. 6, (2007), pp. 553-586.

[11] M. Fantinato, I. M. de S. Gimenes and M. B. F. de Toledo, "Product Line in the Business Process Management Domain”, Applied Software Product Line Engineering, 1st ed., K. C. Kang, V. Sugumaran, and S. Park, Eds. Boca Raton: Auerbach Publications, (2009), pp. 497-530.

[12] J. Van den Bergh, "Promises from SOA: Reengineering a procurement process at Belgacom Mobile - a case study approach”, Business Process Management Journal, vol. 18, no. 5, (2012), pp. 815-828.

[13] Z. Zlatev, "Examination of the negotiation domain”, Enschede, The Netherlands, (2002).

[14] G. Governatori, M. Dumas, A. H. M. ter Hofstede and P. Oaks, “A formal approach to protocols and strategies for (legal) negotiation”, Proceedings of the 8th international conference on Artificial intelligence and law - ICAIL '01, (2001), pp. 168-177.

[15] S. Rinderle and M. Benyoucef, "Towards the automation of e-negotiation processes based on Web services A modeling approach”, Web Information Systems Engineering-WISE 2005 (6th International Conference on Web Information Systems Engineering, New York, NY, USA, Proceedings, LNCS, A. H. H. Ngu, M. Kitsuregawa, E. J. Neuhold, J.-Y. Chung, and Q. Z. Sheng, Eds. New York: Springer Berlin Heidelberg, vol. 3806, (2005) November 20-22, pp. 443-453.

[16] N. R. Jennings, P. Faratin, A. R. Lomuscio, S. Parsons, M. Wooldridge and C. Sierra, "Automated Negotiation: Prospects, Methods and Challenges”, Group Decision and Negotiation, vol. 10, no. 2, (2001), pp. 199-215.

[17] J. B. Kim, A. Segev, A. Patankar and M. G. Cho, "Web Services and BPEL4WS for Dynamic eBusiness Negotiation Processes”, Proceedings of the International Conference on Web Services, (2003), pp. 111-117.

[18] S. Angelov and P. Grefen, "Support for B2B E-Contracting-The Process Perspective", Knowledge and Technology Integration in Production and Services, V. Mařík, L. M. Camarinha-Matos, and H. Afsarmanesh, Eds. Springer US, (2002), pp. 87-96.

[19] S. Angelov and P. Grefen, "An e-contracting reference architecture”, Journal of Systems and Software, vol. 81, no. 11, (2008), pp. 1816-1844.

[20] K. Czarnecki, S. Helsen and U. Eisenecker, "Staged configuration through specialization and multilevel configuration of feature models”, Software Process: Improvement and Practice, vol. 10, no. 2, (2005), pp. 143-169.

[21] M. Fantinato, M. B. F. de Toledo, L. H. Thom, I. M. de S. Gimenes, R. dos S. Rocha and D. Z. Guimarã Garcia, “A survey on reuse in the business process management domain”, International Journal of Business Process Integration and Management, vol. 6, no. 1, (2012), pp. 52.

[22] J. Kim, A. Segev, S. Kim and A. Patankar, "eBusiness System Supporting Collaboration and Negotiation in Design and Procurement of Personalized Solution”, Berkeley, (2003).

[23] G. E. Kersten, S. E. Strecker and K. P. Law, "Protocols for electronic negotiation systems: theoretical foundations and design issues”, Lecture Notes in Computer Science // 5th International Conference, EC-Web 2004, Zaragoza, Spain, Proceedings, EC-Web 200., vol. 3182, K. Bauknecht, M. Bichler, and B. Pröll, Eds. Montreal: Springer Berlin / Heidelberg, (2004) August 31-September 3, pp. 106-115.

[24] S. Wu, G. E. Kersten and M. Benyoncef, "INSS-a new approach in designing Web-based negotiation support systems”, Proceedings of the Montreal Conference on e-Technologies, (2006), pp. 1-15. 
[25] F. G. Marchione, M. Fantinato, M. B. F. de Toledo and I. M. S. Gimenes, "Price definition in the establishment of electronic contracts for web services", Proceedings of the 11th International Conference on Information Integration and Web-based Applications \& Services - iiWAS ’09, (2009), pp. 217.

[26] T. L. Goncalves, I. M. D. S. Gimenes, M. Fantinato, G. H. Travassos and M. B. F. De Toledo, "Experimental studies of e-contract establishment in the PL4BPM context”, International Journal of Web Engineering and Technology, vol. 6, no. 3, (2011), pp. 243.

[27] M. Bichler, G. Kersten and S. Strecker, “Towards a Structured Design of Electronic Negotiations”, Group Decision and Negotiation, vol. 12, no. 4, (2003), pp. 311-335.

[28] M. Comuzzi, K. Kritikos and P. Plebani, "A Semantic Based Framework for Supporting Negotiation in Service Oriented Architectures”, 2009 IEEE Conference on Commerce and Enterprise Computing, (2009), pp. 137-145.

[29] M. Mukhtar, Y. Yahya, N. Jailani, S. Abdullah and Z. Abdullah, “An integrated e-marketplace framework: A case study of wood-based products industry in Malaysia”, 2009 International Conference on Electrical Engineering and Informatics, (2009), pp. 314-320.

[30] D. K. W. Chiu, S. C. Cheung, P. C. K. Hung, S. Y. Y. Chiu and A. K. K. Chung, "Developing e-Negotiation support with a meta-modeling approach in a Web services environment”, Decision Support Systems, vol. 40, no. 1, (2005), pp. 51-69.

[31] A. Elfatatry and P. Layzell, "Negotiating in service-oriented environments", Communications of the ACM, vol. 47, no. 8, (2004), pp. 103-108.

[32] D. A. Vecchiato, M. B. F. Toledo, M. Fantinato and I. M. S. Gimenes, “A Feature-based Toolkit for Electronic Contract Negotiation and Renegotiation", Proceedings of the IADIS International Conference WWW/Internet 2010, (2010), pp. 3-10.

\section{Authors}

Gabriel Costa Silva is a PhD student and member of Enterprise Systems group at the University of York, UK. He received his M.Sc. degree in Computer Science (2010) from State University of Maringá, Brazil. His current research interest includes electronic negotiation, service-oriented computing, and cloud computing.

Itana Maria de Souza Gimenes is a professor of Software Engineering at State University of Maringá, Paraná, Brazil. Post-doctoral research at the School of Computer Science, University of Waterloo, ON, Canada (2005). Ph.D. in Computer Science at the University of York, Department of Computer Science, UK (1992). President of the Brazilian Computer Society Committee of Software Engineering in 2007-2008 and 1998-1999 (CEES-SBC). Has lead several research projects, including international cooperation with the European Community. Current research interests include: software architecture, software product line, component-based development, workflow management systems and business process management.

Marcelo Fantinato is an assistant professor at the University of São Paulo, Brazil. He is Doctor in Computer Science (2007) and Master of Engineering (2002) at the University of Campinas, Brazil. His main research interests are business process management, service-oriented computing, electronic contracts, software product line and software testing.

Maria Beatriz Felgar de Toledo is an associate professor in the Institute of Computing, University of Campinas (UNICAMP), Brazil. She received her M. Sc. in Computer Science from UNICAMP and her Ph. D. degree from Lancaster University, UK (1992). Her main research interests are business process management systems and service-oriented architectures. 
International Journal of $\mathrm{u}$ - and e- Service, Science and Technology Vol.6, No.5 (2013) 